\title{
Experimental Evidence for the Acceleration of Thermal Electrons by Ion Cyclotron Waves in the Magnetosphere
}

\author{
A. J. NORRIS, J. F. E. JOHNSON'1, J. J. SOJKA2², AND G. L. WRENN \\ Mullard Space Science Laboratory, University College London, \\ Holmbury St. Mary, Dorking, Surrey, U.K.
}

N. Cornilleau-Wehrlin, S. Perraut, And A. Roux

Centre de Recherces en Physique de l'Environnement, Centre National d'Etudes des Telecommunications 92131 Issy-les-Moulineaux, France

\begin{abstract}
Ion cyclotron waves (ICWs) at frequencies just above $f_{\mathrm{He}^{+}}$, the helium gyrofrequency, are often observed by the ESA satellites GEOS 1 and GEOS 2. Young et al. (1981) have shown the close connection between these ICWs and the presence of thermal $\mathrm{He}^{+}$ions in the outer magnetosphere. The purpose of this paper is to provide experimental evidence for the acceleration of thermal electrons by the large amplitude ICWs. The wave power in the ultra low frequency (ULF) range near $f_{\mathrm{He}^{+}}$is compared with the distribution function of low energy electrons, measured by instruments aboard the GEOS satellites. It shows that, near the geomagnetic equator, electrons are accelerated along field lines at times when the ICW energy is large and the cold plasma density is below a threshold value. It is suggested that these accelerated electrons can account for the ELF emissions, modulated at the ICWs frequency, observed by CornilleauWehrlin (1981). Other ULF events having frequencies close to the proton gyrofrequency $f_{\mathrm{H}^{+}}$are also observed and result in a very efficient acceleration of thermal electrons along field lines. The evidence in this case is that medium energy protons having large temperature anisotropies $\left(T_{\perp} \gg T_{||}\right)$in the 100 - to $500-\mathrm{eV}$ range are responsible for the ICW wave generation.
\end{abstract}

\section{INTRODUCTION}

Young et. al. [1981] have recently shown that the GEOS satellites often observe ion cyclotron waves (ICWs hereinafter) at 0.2 to $1.5 \mathrm{~Hz}$ with spectra characterized by the $\mathrm{He}^{+}$gyrofrequency $f_{\mathrm{He}^{+}}$. These emissions are associated with an increase in the $\mathrm{He}^{+} / \mathrm{H}^{+}$ratio of the 'cold' ion population of $\sim 7-20 \%$, as measured by the GEOS ion composition experiment (ICE; see Balsiger et al. [1976]) at these times. The fact that the ICWs spectra are influenced by $\mathrm{He}^{+}$ions was also shown by Mauk et al. [1981] using ATS 6 data.

Young et al. [1981] have noted that ICWs observed by the two GEOS spacecraft generally have different characteristics. ICWs observed at GEOS 2, in the equatorial plane, generally have left-hand circular polarization, while those seen by GEOS 1 , at $\sim 15-25^{\circ}$ geomagnetic latitude $\left(\lambda_{m}\right)$, often exhibit a linear polarization. This has been interpreted as a propagation effect; ICWs that are generated near the geomagnetic equator at the expense of energetic protons having $T_{\perp}>T_{||}$, undergo a polarization reversal while they propagate toward higher latitudes. This polarization reversal results from the presence of thermal helium ions but such a dramatic change in polarization characteristics only occurs when their frequency is greater than the helium gyrofrequency

${ }^{1}$ Now at Marshall Space Flight Center, Huntsville, Alabama

${ }^{2}$ Now at Utah State University, Logan, Utah 84322.

Copyright 1983 by the American Geophysical Union.

Paper number 2A1098.

0148-0227/83/002A-1098\$05.00
$\left(f>f_{\mathrm{He}^{+}}\right)$in an equatorial region where the $\mathrm{He}^{+}$density is sufficiently high.

Rauch [1981] has recently used a ray tracing technique to confirm this theory and show that a wave that is initially left-handed in the equatorial plane becomes righthanded or linearly polarized away from it. Then, due to the presence of $\mathrm{He}^{+}$ions, ICWs are reflected when their frequency becomes approximately equal to the local bi-ion hybrid frequency. Since the ICWs are well guided, except very close to the reflection point, they can travel back and forth between the $\mathrm{He}^{+}$induced mirrors on either side of the geomagnetic equator. Roux et al. [1982a] have suggested that these multiple reflections (producing a feedback effect) lead to enhanced ICW amplication, thus explaining the catalytic role played by $\mathrm{He}^{+}$ions in the instability process.

The purpose of this short paper is to give experimental evidence of an acceleration of the electrons by the ULF waves. Data from the Mullard Space Science Laboratory suprathermal plasma analyzer (SPA) onboard GEOS [Wrenn et al., 1981] are presented, and comparisons are made with ULF wave data of the GEOS S-300 experiment [S-300 Experimenters, 1979; Jones, 1978; Perraut et al., 1978]. A few specific cases, taken from both GEOS satellites are described and a statistical perspective is given. The results are interpreted in terms of the acceleration of the electrons by the quasi-electrostatic ICWs.

Cornilleau-Wehrlin [1981] has recently reported a class of electrostatic ELF waves that are modulated at the ICW frequency. She has shown that there is good correlation between the occurrence of these ELF events and ICWs when (1) the ULF power exceeds $0.002 \mathrm{y}^{2} \mathrm{~Hz}^{-1}$, and (2) the cold plasma density is less than $\sim 25 \mathrm{~cm}^{-3}$. 


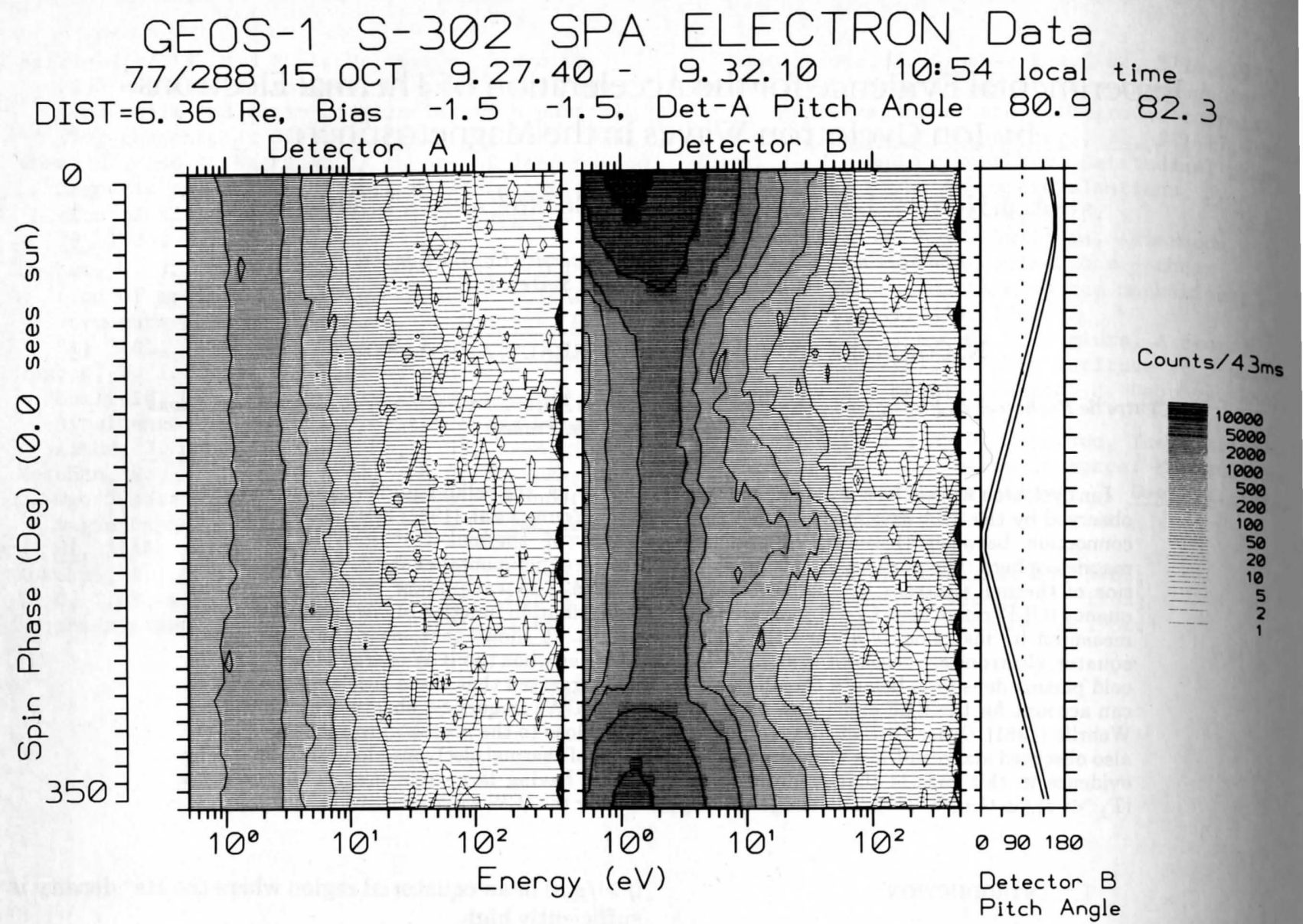

Fig. 1. A contoured grey-scale representation of the SPA count rates as a function of energy and spin phase. The spin phase direction is down the plot, zero phase corresponds to the detector ' $\mathrm{B}$ ' view direction closest to the sun. The nominal $(0.5-500 \mathrm{eV})$ energy scale is indicated, the sensor bias was $-1.5 \mathrm{~V}$. Date, UT range, and local time appear at the top of the frame. At this time the satellite was half inverted, the angle between its spin axis and $\mathbf{B}_{\circ}$ was $81^{\circ}$.

\section{INSTRUMENTATION}

\subsection{Suprathermal Plasma Analyzer (S-302)}

The GEOS SPA consists of two hemispherical electrostatic analyzers mounted on a radial boom, such that detector ' $A$ ' looks parallel to the satellite spin axis and ' $\mathrm{B}$ ' views at $80^{\circ}$ to this. Pitch angle sampling depends on the satellite spin and the orientation of the geomagnetic field $B_{\circ}$. Both analyzers detect electrons or ions in the energy range $0.5-500 \mathrm{eV}$ using 64 channels, each with $11 \%$ energy resolution. The whole sensor unit can be biassed from $-28 \mathrm{~V}$ to $+34 \mathrm{~V}$ with respect to the spacecraft ground in order to overcome floating potential barriers and increase sensitivity for cold particles. A full spectrum can be collected in $2.8 \mathrm{~s}$, but the need for mode and energy range switching limits temporal resolution; for a detailed description of the experiment, see Wrenn et al. [1981].

The SPA data are frequently modulated as a function of spin phase due to a number of effects, and the type of modulation may vary for different energies. In order to assess such complexities rapidly, the data are sorted in phase and energy for both detectors for a single mode (proton or electron) and then the resulting matrix is greyscaled as in Figure 1 [Johnson, 1981]. Since the magnetic field direction here is $81^{\circ}$ from the spin axis, detector ' $\mathrm{A}$ ' samples such a pitch angle but detector ' $B$ ' scans almost all pitch angles during the satellite rotation as shown by the right hand panel. The low-energy features at the top and bottom of the frame are locally produced photoelectrons that dominate on the sunward side of the satellite. Between 60 and $200 \mathrm{eV}$ a weak 'pancake' [Wrenn et al, 1979] distribution appears with detector ' $B$ ' counts maximizing for $90^{\circ}$ pitch angles but below $60 \mathrm{eV}$ in the center of the ' $\mathrm{B}$ ' frame, the field-aligned suprathermal component is clearly visible as it emerges from the photo electron background. In normal attitude the field direction lies close to the spin axis and field-aligned fluxes are measured by detector 'A'.

\subsection{Wave Experiment (S-300)}

For a detailed description of the GEOS wave experiment see Jones [1978] and S-300 Experimenters [1979]. Here we are mainly concerned with the ULF waves $(0.1<f<11 \mathrm{~Hz})$. The data come from three magnetic antennae $B_{x}, B_{y}, B_{z}$, with $B_{z}$ being directed along the satellite spin axis and approximately parallel to the magnetic field for much of the time. In order to remove the effects of the satellite spin, the $B_{x}$ and $B_{y}$ components 


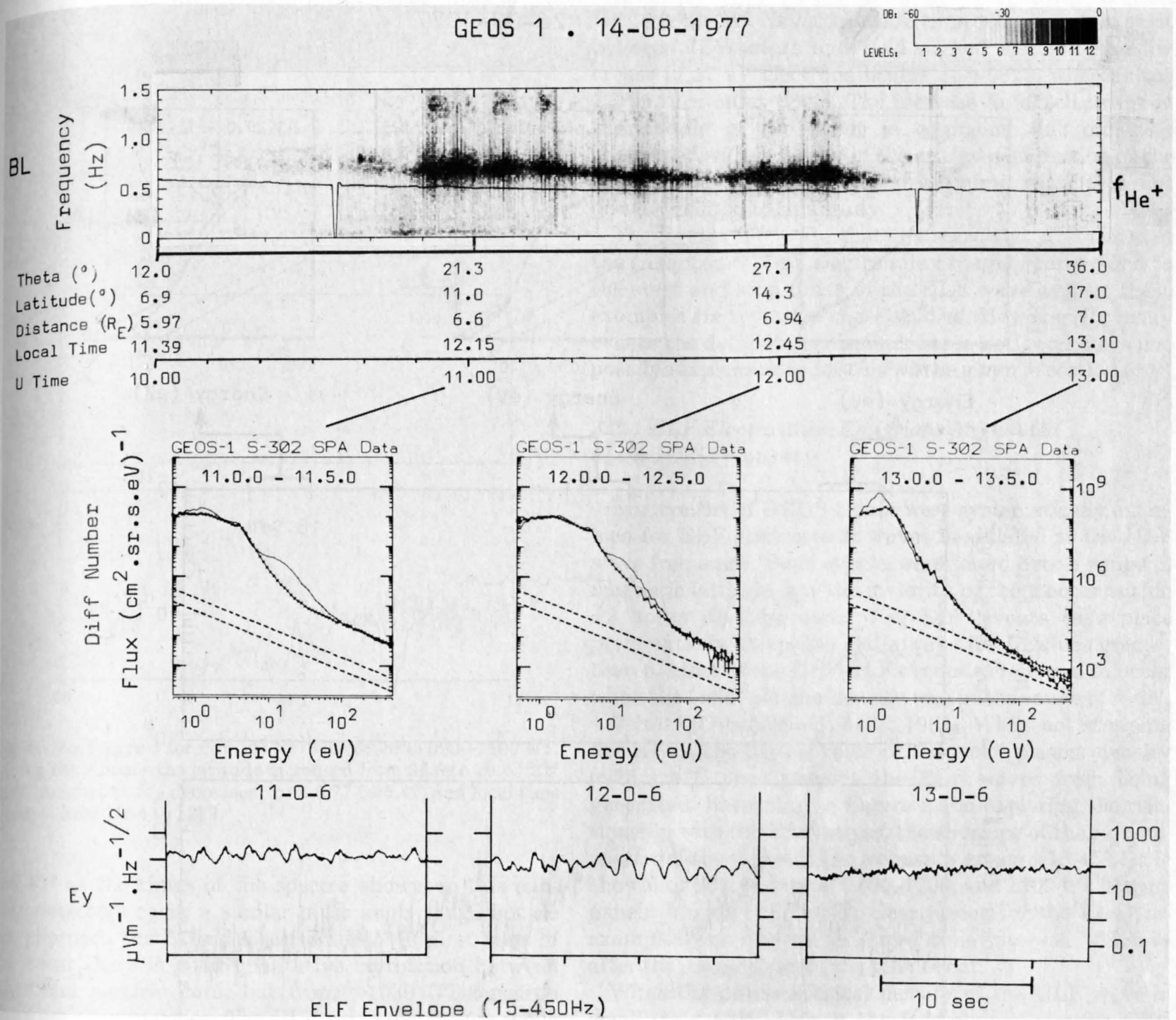

Fig. 2. The top of the figure shows a grey-scale spectogram of the $B_{L}$ component of the ULF data for day 1977/226 (August 14) 1000-1300 UT. The middle three panels show electron differential number flux spectra each of 5 min integration at 1100, 1200 , and 1300 UT respectively for both detectors ' $\mathrm{A}$ ' and 'B' $(x)$; one count levels are included for comparison (sensor bias $=0.0 \mathrm{~V})$. The lower three panels show the envelope of the $E_{y}$ component of the ELF signal for $\sim 20 \mathrm{~s}$ of data at 1100,1200 , and 1300 UT, respectively.

are processed to give $B_{L}$ and $B_{R}$ the circularly polarized components of the ULF signals in the plane perpendicular to the spin axis (left and right-handed, respectively). Data in the ELF range $(15-450 \mathrm{~Hz})$ are also available from the same magnetic sensors and sometimes from the long rgdial boom $E_{y}$ sensor.

The data from this experiment are conveniently presented as grey-scaled spectograms of the $B_{L}, B_{R}$, or $B_{z}$ components. Figure 2 includes a panel for $B_{L}$ covering the frequency range of $0-1.5 \mathrm{~Hz}$, the grey level key shows intensities referred to 0.90 gamma $^{2}$ per $\mathrm{Hz}\left(1_{\gamma}=1 \mathrm{nT}\right)$. The helium gyrofrequency $f_{\mathrm{He}^{+}}$is also plotted on the panel and clearly the ICW power is concentrated just above $f_{\mathrm{He}^{+}}$throughout the event as shown by Young et al. [1981]. In order to display temporal variations it is better to use the integrated power $\left(y^{2}\right)$ of the wave components and in Figures 3 to 5 the power in $B_{L}$ between 0.32 and $1.5 \mathrm{~Hz}$ (eliminating any spin frequency contamination at $\sim 0.17 \mathrm{~Hz}$ ) is plotted on a logarithmic scale. On
GEOS 1 the $B_{R}$ component was, in general, insufficiently different from $B_{L}$ to warrant plotting it separately.

\section{ObServations}

3.1. Evidence for the Acceleration of Field-Aligned Electrons and Their Correlation with ICWs Having $f \sim f_{\mathrm{He}^{+}}$

In a search through the SPA data at the time of ICW events observed by the S- 300 wave experiment, a consistent enhancement is observed in the low energy electrons provided that the SPA is in a suitable mode. Owing to the mode scheduling of both experiments a statistical study is not easy to do, although it is feasible with GEOS 2. For some events it is possible to compare the evolution of the $\sim 10-20 \mathrm{eV}$ enhanced electron flux and the spectral power density of the ULF waves. Figure 2 presents a typical event seen by GEOS 1 with strong enhancements of ULF emissions above the helium gyrofrequency for well over 

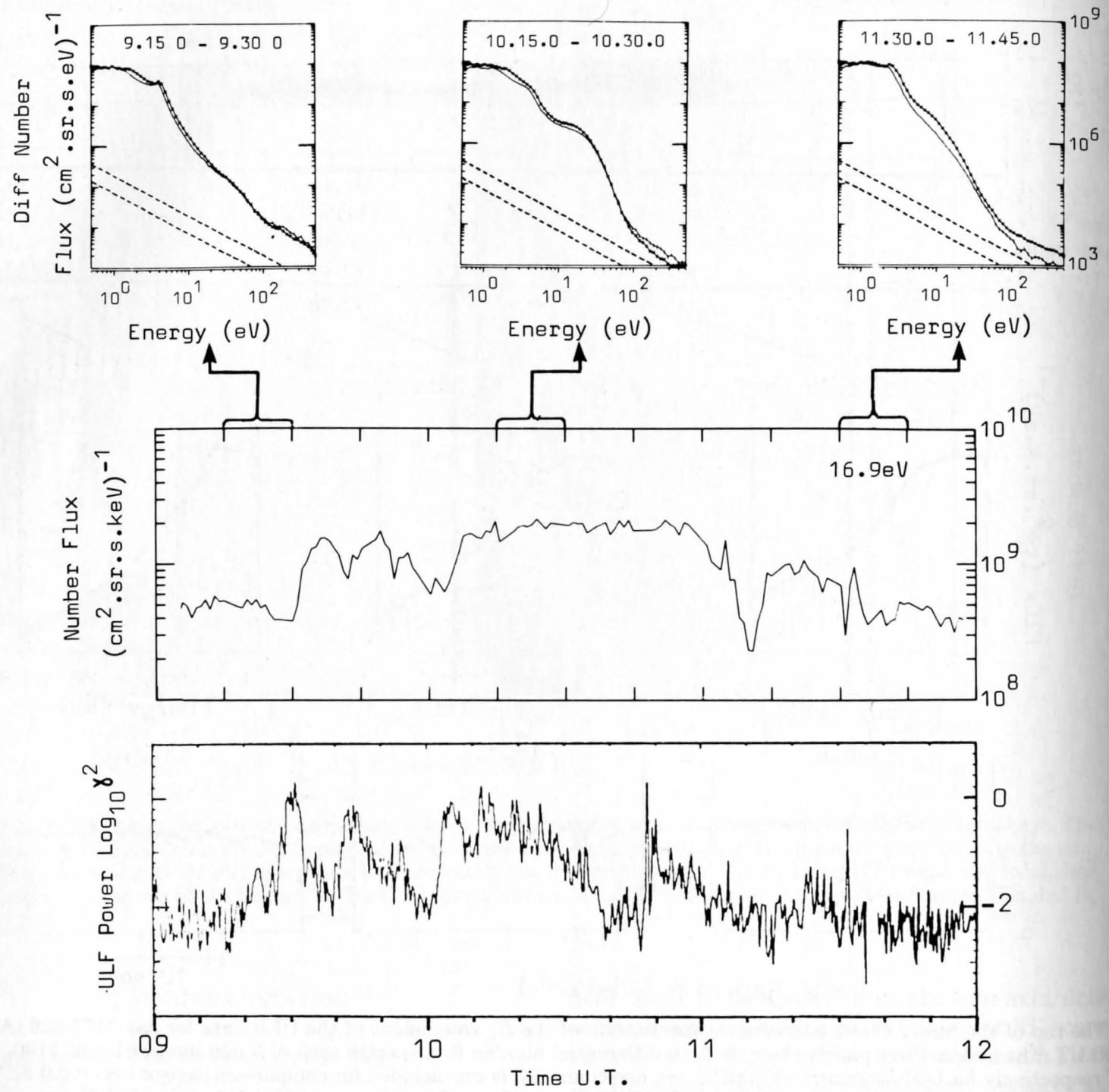

Fig. 3. At the top of the figure are three pairs of electron differential number flux spectra each of 15 min integration, the start time is indicated by an arrow from the panel below, which shows the time evolution of the $\sim 17 \mathrm{eV}$ electron differential number flux for detector 'A' (sensor bias $=-1.5 \mathrm{~V}$ ). In the lower panel is the ULF power in $B_{L}$ 0.30-1.5 Hz. During the 3 hours the latitude increased from $20.8^{\circ}$ to $25.9^{\circ}\left(19^{\circ}\right.$ to $28^{\circ}$ magnetic), the geocentric distance decreased from 6.96 to $5.92 R_{E}$, and the local time changed from 1035 to 1226 .

an hour on August 14th (day 226) 1977. The extent of latitude, geocentric distance, and local time is noted together with theta, the angle between the spin axis, and $\mathbf{B}_{\circ}$. The geomagnetic latitude, determined from a model field, increased from $4^{\circ}$ to $18^{\circ}$ during the three hours. Low-energy electron spectra are only available every 30 min in this period but the three examples selected do show that electron flux between $7 \mathrm{eV}$ and $100 \mathrm{eV}$ at small pitch angle (detector ' $A$ ', thin line) is increased during the strong wave activity between 1050 and 1210 UT. The detector ' $A$ ' pitch angle ranges from $21^{\circ}$ at 1100 UT to $27^{\circ}$ at $1200 \mathrm{UT}$ and $36^{\circ}$ at $1300 \mathrm{UT}$, while detector 'B' covers 60 to $90^{\circ}$. The lack of suprathermal electrons on detector ' $A$ ' at 1300 UT is probably linked to the end of the ICW event but it could also relate to the changing direction of $\mathbf{B}_{\circ}$. The ELF measurements (bottom panel) for this event will be discussed later.

A survey of the SPA data indicated that examination of a single energy channel at $16.9 \mathrm{eV}$ would yield a suitable temporal profile for comparison with the ULF measurements that could be quantified in terms of an integrated power. Figures 3,4 , and 5 give examples of this procedure.

The event shown in Figure 3 is for September 26 (day 269 ), 1977, starting at $\sim 0935$ UT and finishing at $\sim 1130$ UT. The three electron spectra taken before, in the middle, and at the end of the event exhibit the appearance and decay of the characteristic suprathermal bulge also reflected in the $17 \mathrm{eV}$ channel monitor of the second panel. Theta is large through this period, being $42^{\circ}, 49^{\circ}$, 
GEOS $1 \cdot 6-10-1977$
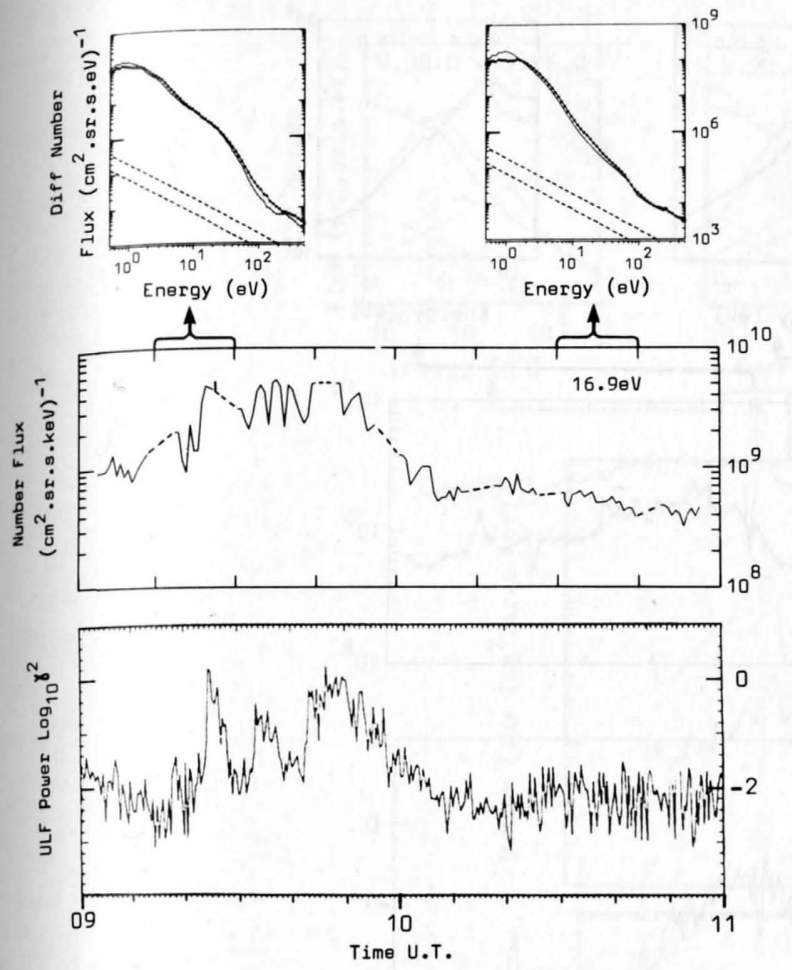

Fig. 4. As Figure 3 for day $1977 / 279$ (October 6) 0900-1100 UT. During the 2 hours the latitude increased from 24.6 to $26.4^{\circ}\left(22^{\circ}\right.$ to $26^{\circ}$ magnetic), $R_{E}$ decreased from 6.77 to 5.42 , and local time changed from 1054 to 1222 .

and $61^{\circ}$ at the times of the spectra shown; in this case both detectors cover a similar pitch angle range but do not approach the B field direction. For the first hour of the event there is good qualitative correlation between wave and particle data, but from 1030 UT onwards there are discrepancies. The ULF wave disappears while the electron enhancement remains; note that here the magnetic latitude is increasing above $25^{\circ}$.

Figure 4 shows similar data for October 6 (day 279), 1977. A significant density enhancement of the suprathermal electrons is found, with fluctuations on a time scale of $\sim 3 \mathrm{~min}$. These fluctuations correlate well with the enhanced magnetic signals from 0.3 to $1.5 \mathrm{~Hz}$ observed simultaneously. The spectral modification is less spectacular in this event but unmistakeable nevertheless. The low-energy fluctuations are not due to pitch angle changes, since theta varies only slowly between $35^{\circ}$ and $45^{\circ}$ during this period; again field-aligned measurements are not available.

GEOS 2 staying close to the geomagnetic equator, has a better chance of giving data at low pitch angle: the event on November 17 (day 321), 1978, shown in Figure 5 lasts from $\sim 0800-1020 \mathrm{UT}$; theta remains at $12 \pm 2^{\circ}$ throughout and here data from both detectors are plotted. There is initially a 'pancake' distribution at the higher energies; but this weakens through the event. The enhancement in the field-aligned electron component can be clearly seen, superimposed as it is on the background 'pancake'. Correlation between the low-energy electrons and ULF signals is good as witnessed by the $17 \mathrm{eV}$ profile for detector ' $A$ '. Nevertheless, a time delay seems to exist between ICW start and field-aligned electron rise (increase in $17 \mathrm{eV}$ electrons begins at $\sim 0820$, whereas the ICWs turn on at 0805). The increase in $90^{\circ}$ electrons in the middle of the event is confusing but must be associated with a change in the energy distribution of the background 'pancake', and we assume that it is not directly related to this study.

The figures illustrate that the start and stop times of the enhanced $\sim 17-\mathrm{eV}$ electron flux roughly correspond to the start and stop times of the ULF wave events, these examples are typical of those studied. However, for many events the detailed correspondence is not good but some possible explanations for this will be given in section 5 .

\subsection{ELF Electrostatic Emissions Modulated at the ICW Frequency}

Six months of GEOS 1 data were systematically examined for ELF electrostatic waves modulated at the ULF wave frequency. Such events were found over a range of magnetic latitude, but the majority of them occur within \pm 2 hours of local noon. The ELF events take place preferentially at apogee and always for $L$ values greater than 6. Most of the ULF-ELF events were found to occur when the 'cold' plasma density was in the range of $\sim<N_{e}$ $<7 \mathrm{~cm}^{-3}$ [Cornilleau-Wehrlin, 1981]. While not stopping the ICWs, too high a value of the 'cold' plasma density ( $\sim 25 \mathrm{~cm}^{-3}$ ) does prevent the ELF waves from being generated. Returning to Figure 2 and exploring the relationship with the ELF waves, the envelope of the $E_{y}$ component of the signal in the frequency range $\sim 15-450 \mathrm{~Hz}$ is shown for $20 \mathrm{~s}$ of data at 1100,1200, and 1300 UT (lowest panel). Modulation can be clearly seen for the first two examples but not for the third time interval, which is after the termination of the ICW event.

When the power spectral density of the ULF wave is small $\left(B^{2}<0.05 \gamma^{2} \mathrm{~Hz}^{-1}\right)$ the field-aligned electrons are not observed, neither are the ELF associated waves. This might also account for the lack of correlation between ICWs and field-aligned electrons at the beginning of the event shown in Figure 5.

\subsection{Relationship Between $f \sim f_{\mathrm{H}^{+}} I C W s$ and Suprathermal Particles}

Another interesting class of ULF wave events have been observed, these have a frequency very close to, but slightly less than the proton gyrofrequency $f_{\mathrm{H}^{+}}$. For such an event on August 5 (day 217), 1977, 0920-0940 UT, there is a particularly strong parallel acceleration of the electrons (see Figure 6), and theta is steady between 5 and $10^{\circ}$. With such a small theta, detector ' $A$ ' samples the 'loss' cone and collects a background of photoelectrons of ionospheric origin, but this does not mask the enhancement at $\sim 17 \mathrm{eV}$ in the electron spectra nor the good correlation with the power of ULF waves perpendicular to $B_{\text {oin }}$ the frequency range 1.5 to $5 \mathrm{~Hz}$, plotted in the lower panel.

The medium energy protons $(10-100 \mathrm{keV})$ that usually provide energy to the ICWs [Young et al., 1981] cannot cyclotron resonate with these waves which have a nor- 
GEOS $2 \cdot 17-11-1978$
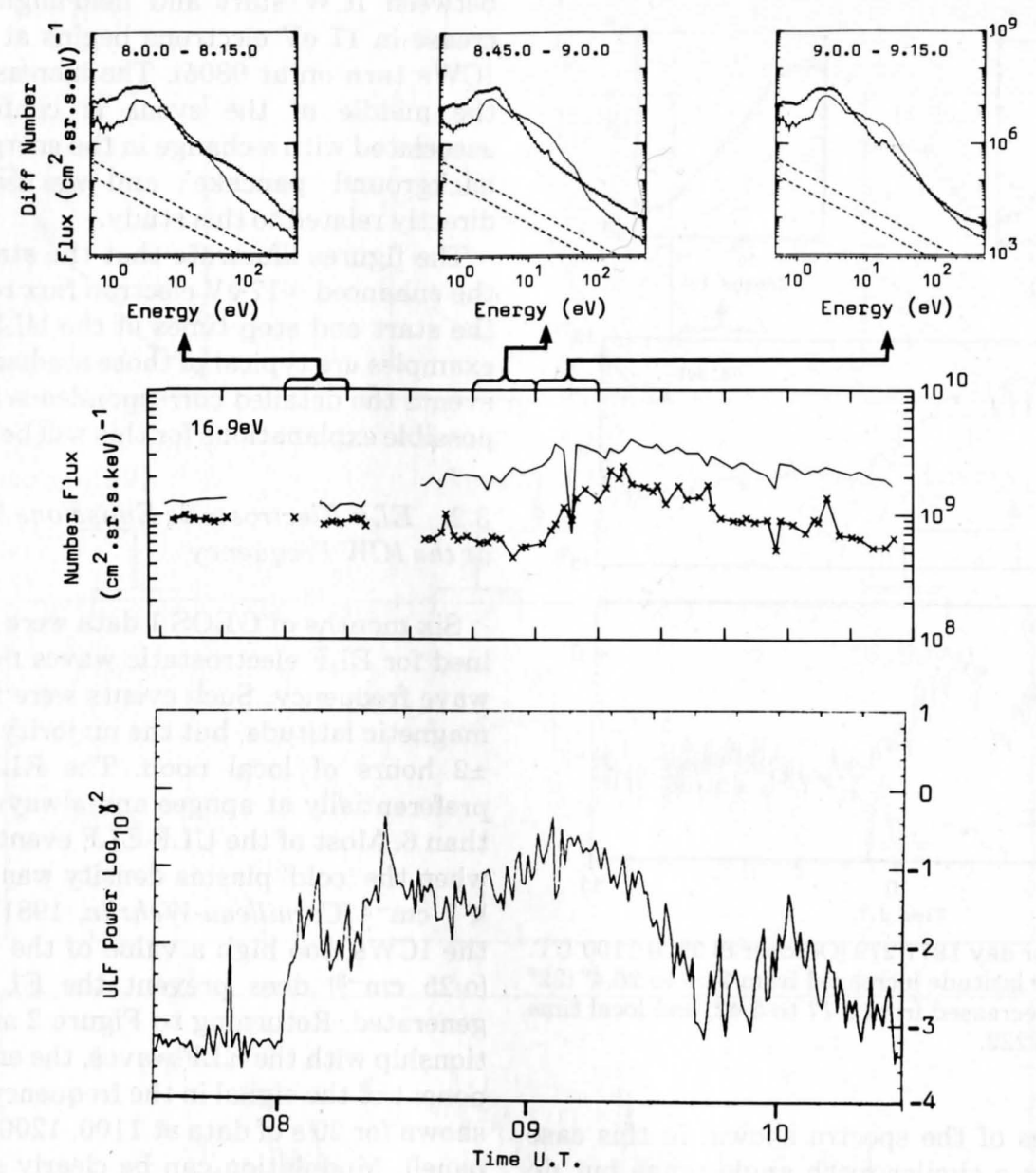

Fig. 5. As Figure 3 for day 1978/321 (November 17) 0730-1030 UT but plotting the flux for both detectors (sensor bias $=-4.0 \mathrm{~V}$ ) and the $B_{R}$ component of ULF. During the 3 hours the latitude increased from -0.6 to $-0.3^{\circ}$ $\left(-4^{\circ}\right.$ magnetic), the local time was UT +2.5 hours.

malized frequency $X=f / f_{\mathrm{H}^{+}}$close to unity. In this case, the Doppler shift $k_{\|} \cdot v_{\|}$in the resonance condition must be small; reasonable values of $k_{\|}\left(k_{\|} \sim w / V_{A}, V_{A}\right.$ being the Alfven velocity) determine that only low-energy protons with large anisotropies, $T_{\perp} / T_{\|}>(1-X)^{-1} \gg 1$, can destabilize the ICW. However, Figure 7 shows that strong pitch angle anisotropy $\left(T_{\perp} \gg T_{\| \mid}\right)$occurs in the low-energy protons $(\mathrm{E} \sim 100-500 \mathrm{eV})$, and the waves could well be driven by such a source of free energy. In addition to this strong pitch angle anisotropy, the ion flux over the entire SPA energy range exhibits an approximately 4-min modulation that puts it in the PC-5 range. The time variation of the ion flux at its peak energy $(E \sim 100 \mathrm{eV})$ is plotted in Figure 8; the same modulation is evident in the wave amplitude.

\section{Statistical Study}

For two months (November and December 1978), we have listed ULF PC-1 type wave events (see Table 1) observed by GEOS 2 that satisfy both of the following criteria:

1. Their frequency lies in the range $f_{\mathrm{He}}+<f<f_{\mathrm{H}^{+}}$, (this will be justified in the next section).
2. They have a magnetic power $b^{2}>0.1 \gamma^{2}$.

The $\sim 17-\mathrm{eV}$ channel electron count rate from the parallel detector ('A') for November and December 1978 (days 305 to 365 ) was plotted as a function of UT and day number, to show the frequency with which such lowenergy enhancements occur. The data were restricted to pitch angles within $\pm 15^{\circ}$ of the field-aligned direction.

Provided that theta is small, detector ' $A$ ' views the field-aligned direction while detector ' $B$ ' scans a range of pitch angles $\left(80^{\circ} \pm \theta\right)$.

The complex mode scheduling of the SPA experiment hinders any attempt at performing a statistical correla. tion with the ULF data set.

However, out of 32 such events we have studied, a number of common features have emerged. For sixteen of these the SPA is not in a suitable mode and/or does not have the required pitch angle coverage. Of all the remaining events, we find that there are only three examples where the ICW emissions are observed without simultaneous low-energy 'field-aligned' electron flux enhancements, in each case the cold plasma density is high.

The characteristic features of the ICWs in the $\sim 17-\mathrm{eV}$ electron data become more pronounced with increasing 


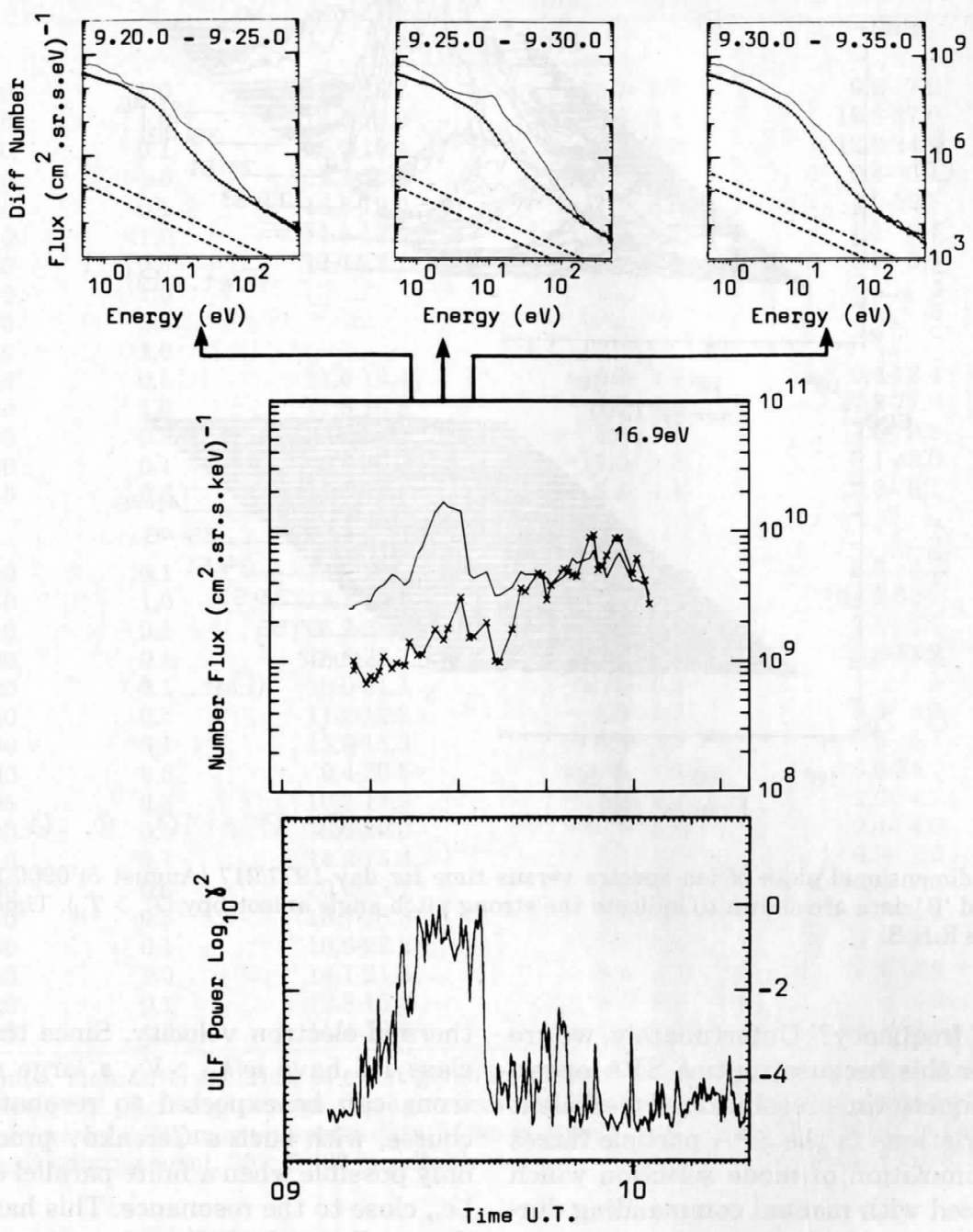

Fig. 6. Data for day 1977/217 (August 5) 0900-1000 UT in a similar format, here the ULF power is for $B_{\text {PERP }}$ $1.5-5 \mathrm{~Hz}$. The SPA sensor bias was $0.0 \mathrm{~V}$. Between 0900 and $1000 \mathrm{UT}$ the latitude increased from -4.3 to $2.8^{\circ}\left(-8^{\circ}\right.$ to $-1^{\circ}$ magnetic), $R_{E}$ increased from 4.28 to 5.50 , and the local time changed from 1052 to 1149 .

wave power. Those events that have $b^{2}>0.3 r^{2}$ are the most easily distinguished from the data plotted. For example, the maximum magnetic power for the event of November 17 (day 321), 1978, 0820-1050 UT (shown in Figure 4) is $0.5 \gamma^{2}$.

This correlation between wave and particle data is true for both of the GEOS satellites. However, for the GEOS 1 events, particularly those of long duration, the detailed correspondence between the two data sets decreases with increasing magnetic latitude.

\section{Discussion}

Simultaneously with the presence of ICWs having $f>$ $\mathrm{H}_{\mathrm{He}}+\mathrm{t}$, one can observe an acceleration of thermal electrons with a clear bias in the $\mathrm{B}_{\circ}$ direction. Mauk and McPherron [1980] have observed the acceleration of thermal electrons in the presence of ICWs using ATS 6. Of particular interest is their Figure 5, which shows time profiles of electron data samples at $30 \mathrm{eV}$ during a wave event.
Using the theory of Stix [1957], they infer that as the wave approaches the ion cyclotron frequency, the motion of the electrons perpendicular to $\mathbf{B}_{\circ}$ does not match that of the ions and results in large perpendicular currents; this condition can generate space charge for nonzero propagation angles. What is actually observed is that the

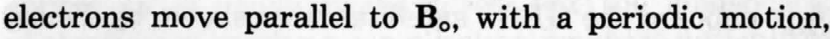
cancelling out the space charge as it is generated and producing a field-aligned current. At much higher frequency in the ELF range, Cornilleau-Wehrlin [1981] has reported observations of electrostatic emissions above the lower hybrid frequency; these ELF waves were shown to be modulated at the ICWs frequency. When intense ICWs are present, thermal electrons are accelerated along field lines and modulated ELF noise does appear as illustrated by Figure 2. This suggests that modulated ELF noise is generated by accelerated electrons. If so, the accelerated electrons should also be modulated at the ICW frequency. Any search for an interpretation then immediately raises the question 'Is the enhanced low-energy electron flux 


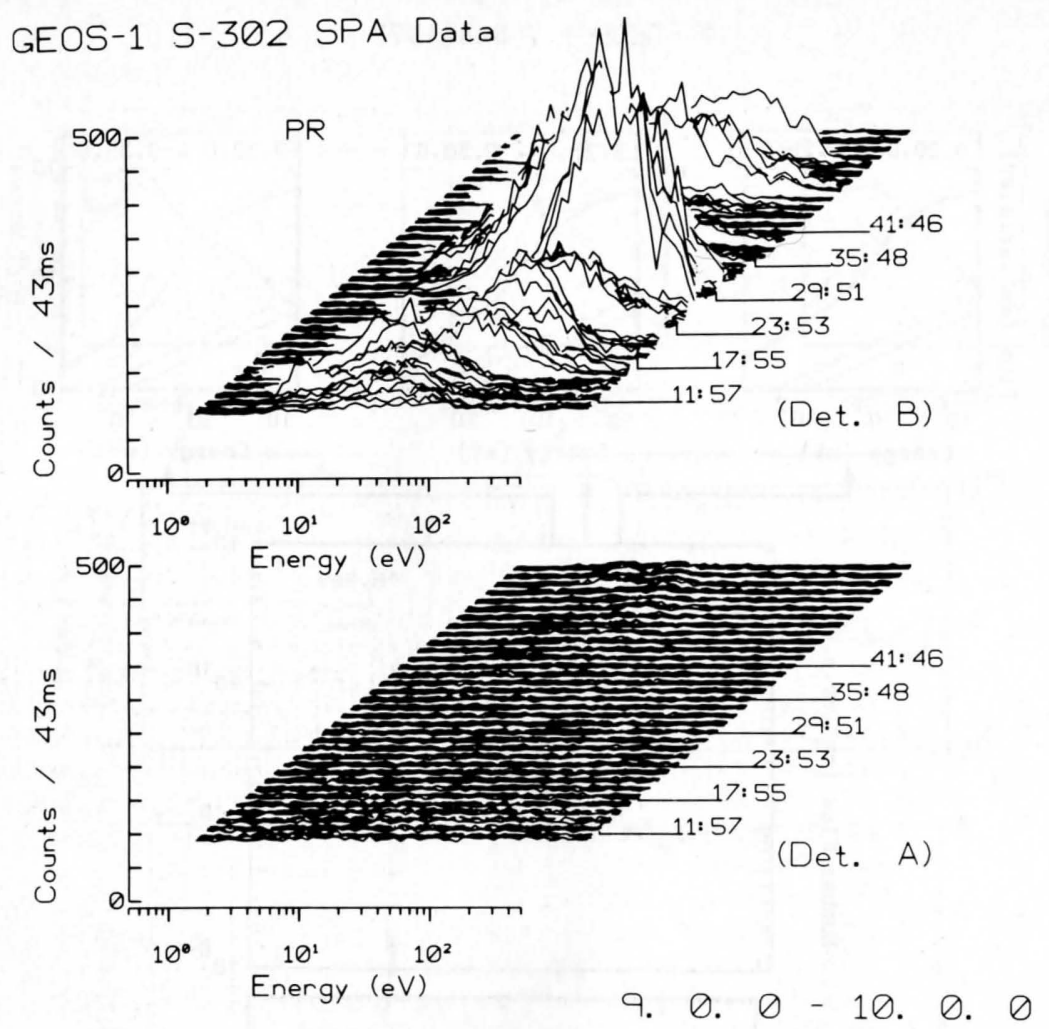

Fig. 7. Three dimensional plots of ion spectra versus time for day 1977/217 (August 5) 0900-1000 UT. Both detector ' $\mathrm{A}$ ' and 'B' data are shown to indicate the strong pitch angle anisotropy $\left(T_{\perp}>T_{\|}\right)$. Times (min:sec) are indicated on the R.H.S.

modulated at the ULF frequency?' Unfortunately, we are not yet able to answer this because routine SPA operation modes have inadequate time resolution. An efficient search for periodic variations in the SPA particle fluxes really requires an optimization of mode selection which could only be guaranteed with manual commanding during real time operations.

The presence of 'heavy' ions in a proton plasma, even in small concentration and with low temperatures $(<1 \mathrm{eV})$, greatly modifies the propagation and amplication characteristics of magnetospheric electromagnetic waves. New resonances and cutoffs appear [Stix, 1962], the wave group velocity is significantly altered and strong coupling may occur between different wave modes [Smith and Brice, 1964; Jones, 1972; Leer et al., 1978].

In an $\mathrm{He}^{+}$rich magnetoplasma the dispersion relation has three branches below $f_{\mathrm{H}^{+}}$. ICWs observed just above $f_{\mathrm{He}^{+}}$onboard the GEOS satellites were shown by Young et al. [1981] to belong to the branch that runs from 0 to a resonance frequency $f_{\text {res }} ; f_{\text {res }}=f_{\text {bi-ion }}$ for $\theta=\left(\mathbf{k}, \mathbf{B}_{\circ}\right)=\pi / 2$, while $f_{\text {res }}=f_{\mathrm{H}^{+}}$for $\theta=0$. This branch is designated as class III in the works of Rauch [1981] and Roux et al. [1982b]. The main characteristics of this branch is that $\mathbf{k}$, the wave vector, is contained in the $\mathbf{E}, \mathbf{B}_{\circ}$ plane. This is linked to the existence of an oblique resonance and ray tracing studies performed by Rauch [1981] indeed show that ICWs approach this resonance. Roux et al. [1982b] will discuss the possible consequences of this process, but it is important to emphasize that for typical values of the cold plasma density in the dayside at $L \sim 6$ to $7 R_{E}$ (e.g., $N_{\text {cold }} \sim 5$ to $10 \mathrm{~cm}^{-3}$ ), the Alfven velocity is just above the thermal electron velocity. Since the ICWs belonging to class III have $w / k_{\|} \sim V_{\mathrm{A}}$ a large number of these electrons can be expected to resonate with the ICW. Of course, with such a Cerenkov process $\left(w / k_{\|} \sim v_{\|}\right)$it is only possible when a finite parallel electric field develops, i.e., close to the resonance. This has been pointed out by Cornwall et al. [1971].

An analysis of the SPA data for a number of these ICW events shows that an enhanced flux of low-energy electrons at small pitch angles is consistently observed. Because detector ' $\mathrm{A}$ ' does not always view in the field-

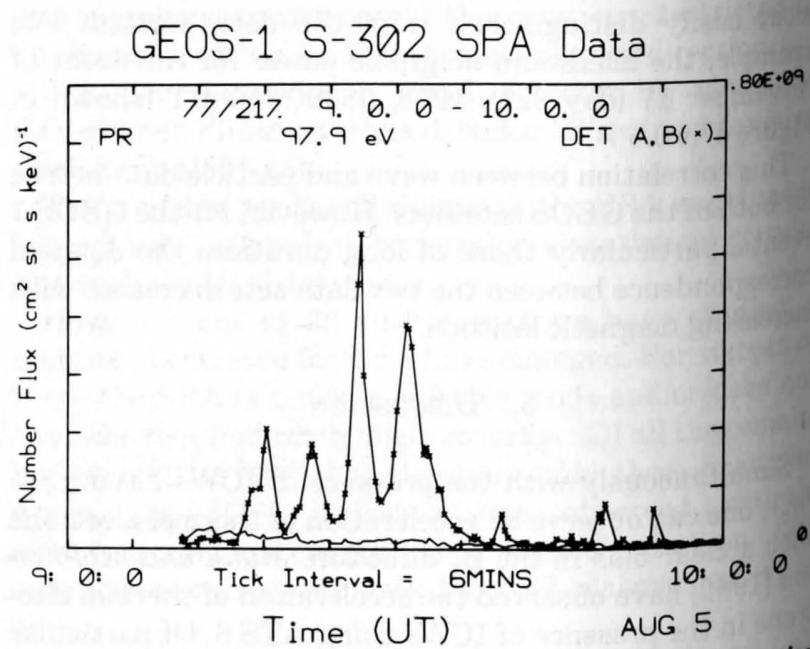

Fig. 8. Differential number flux for $\sim 100 \mathrm{eV}$ ions as a function of time for day 1977/217 (August 5) 0900-1000 UT. 
Table 1. Intense ULF Events $f>f_{\mathrm{He}^{+}}\left(\right.$For $\left.b^{2}{ }_{\max }>0.1 r^{2}\right)$

\begin{tabular}{|c|c|c|c|c|c|c|c|}
\hline \multirow{2}{*}{ Day } & \multirow{2}{*}{$\begin{array}{c}\text { Time Interval, } \\
\text { UT }\end{array}$} & \multirow[t]{2}{*}{$b^{2}, r^{2}$} & \multirow{2}{*}{ PAA Range, deg } & BLAT, deg & \multirow{2}{*}{ Cold Plasma, $\mathrm{cm}^{-3}$} & \multirow[b]{2}{*}{302} & \multirow[b]{2}{*}{ FA } \\
\hline & & & & Min Max & & & \\
\hline \multicolumn{8}{|c|}{ November 1978} \\
\hline & 0920-1030 & 1.0 & $13.6-25.7$ & $-12.7-5.7$ & $0.2-7.2$ & $\mathrm{x}$ & $\mathrm{x}$ \\
\hline 01 & $1100-1130$ & 1.0 & $16.5-18.9$ & $-8.6-7.4$ & $18.8-27.0$ & $\mathrm{x}$ & $\mathrm{x}$ \\
\hline 01 & $1200-1300$ & 0.1 & $15.9-19.4$ & $-8.7 \cdot 6.9$ & $13.9-24.9$ & $\mathrm{x}$ & $\mathrm{x}$ \\
\hline 02 & $0850-1010$ & $>1.0$ & $12.7-15.5$ & $-6.0-4.7$ & 0.4- 3.4 & $r$ & $r$ \\
\hline 06 & $1550-1620$ & 0.1 & $16.8-19.0$ & $-7.7-6.5$ & $4.0-19.8$ & $\mathrm{x}$ & $\mathrm{x}$ \\
\hline 07 & 0900-0930 & $<1.0$ & $11.8-13.2$ & $-4.6-4.1$ & $1.1-6.4$ & $r$ & $r$ \\
\hline $\begin{array}{l}017 \\
17\end{array}$ & $0850-1050$ & 0.5 & $11.1-13.1$ & $-5.0-4.1$ & $0.3-3.6$ & $r$ & $r$ \\
\hline 19 & $0040-0300$ & 1.0 & $\ldots$ & $\ldots$ & $\ldots$ & $\mathrm{x}$ & $x$ \\
\hline 19 & $0840-1300$ & 2.0 & $\ldots$ & $\ldots$ & $\ldots$ & $\mathbf{x}$ & $\mathrm{x}$ \\
\hline 19 & $2300-0210$ & 1.0 & $\ldots$ & $\ldots$ & $\ldots$ & $\mathrm{x}$ & $\mathrm{x}$ \\
\hline 20 & $0420-0620$ & 0.5 & $11.6-19.4$ & $-6.2-3.4$ & $0.4-12.4$ & 2 & r \\
\hline 20 & $1010-1140$ & 1.0 & $11.5-16.9$ & $-7.0-4.2$ & $27.2-77.8$ & $r$ & $r$ \\
\hline 22 & 0910-1040 & 0.5 & $10.2-14.5$ & $-5.9-3.7$ & 1.6- 3.8 & $r$ & $r$ \\
\hline 25 & $0810-1130$ & 0.1 & $8.5-30.2$ & $-15.0-3.3$ & $0.1-43.0$ & $r$ & $r x$ \\
\hline 26 & $1130-1215$ & 0.5 & $13.7-19.2$ & $-8.3-4.4$ & 3.3- 6.1 & $\mathrm{x}$ & $\mathrm{x}$ \\
\hline \multicolumn{8}{|c|}{ December 1978} \\
\hline 01 & $1100-1200$ & 0.1 & $13.7-19.7$ & $-7.7-5.1$ & $0.5-3.7$ & $\mathbf{x}$ & $\mathbf{x}$ \\
\hline 01 & $1210-1240$ & 1.0 & $13.1-18.9$ & $-7.2-5.0$ & $34.1-62.2$ & $\mathbf{x}$ & $\mathrm{x}$ \\
\hline 02 & $2300-2310$ & 0.1 & $25.9-28.5$ & $-15.2-13.7$ & $3.1-3.1$ & $\mathbf{x}$ & $\mathrm{x}$ \\
\hline 03 & $1710-1800$ & 0.1 & 18.8-23.7 & $-10.5-6.8$ & $8.8-13.2$ & $\mathbf{x}$ & $\mathrm{x}$ \\
\hline 04 & 0100-0400 & 0.1 & $18.0-31.1$ & $-15.5-6.2$ & $\ldots$ & $\mathrm{x}$ & $\mathrm{x}$ \\
\hline 04 & $1115-1140$ & 0.1 & $11.2-12.3$ & $-3.9-3.3$ & $0.9-3.0$ & $r$ & $r$ \\
\hline 04 & $1300-1400$ & 0.1 & $13.0-15.3$ & $-4.9-3.7$ & $0.8-5.1$ & $r$ & $r$ \\
\hline 14 & $0900-1200$ & 0.5 & $9.4-20.5$ & $-9.0-0.2$ & $0.6-34.7$ & $r$ & $r x$ \\
\hline 16 & $1340-1405$ & 0.3 & $10.7-11.9$ & $-5.0-4.4$ & $2.0-4.7$ & $r$ & $r$ \\
\hline 16 & $1600-1700$ & 0.1 & $12.6-20.0$ & $-10.9-6.4$ & 2.4- 4.0 & $r$ & $?$ \\
\hline 17 & $0850-1010$ & 0.1 & $14.2-15.4$ & $-5.7 \cdot 5.0$ & $0.3-1.5$ & $r$ & $r$ \\
\hline 17 & $1250-1600$ & 0.5 & $10.6-13.7$ & $-6.2-4.4$ & 2.0- 7.3 & $r$ & $r$ \\
\hline 21 & $1150-1220$ & 0.5 & $15.7-18.8$ & $-8.2-6.5$ & $1.7-6.8$ & $\mathrm{x}$ & $\mathrm{x}$ \\
\hline 22 & $0450-0530$ & 0.1 & $18.6-22.2$ & $-7.6-5.3$ & $0.1-0.4$ & $\mathrm{x}$ & $\mathrm{x}$ \\
\hline 22 & $1120-1200$ & 1.0 & $14.1-21.3$ & $-9.4-5.7$ & 2.3- 4.6 & $\mathrm{x}$ & $\mathrm{x}$ \\
\hline 29 & $0930-1020$ & 0.1 & $12.8-15.3$ & $-6.1 \cdot 4.0$ & $0.7-1.2$ & $r$ & $r$ \\
\hline 29 & $1100-1230$ & 1.0 & $11.4-13.6$ & $-6.1 \cdot 3.1$ & $0.9-2.8$ & $r$ & $r$ \\
\hline
\end{tabular}

Key. Day: Day of month. Time Interval: Time of event given in the form hour:minute. $b^{2}$ : The maximum magnetic power of the wave. PAA Range: Detector ' $A$ ' pitch angle range through event. BLAT: Range of effective magnetic latitude sampled through event. This is calculated from the S-331 magnetometer data. If we assume a dipole field, then BLAT $=\operatorname{Tan}^{-1}[-V / 2 H]$. Cold Plasma: Range of thermal ion density during event. 302: S-302 in suitable mode, $r=$ yes, $x=$ no. FA: Field-aligned flux, $r=$ present, $x=$ not present.

aligned direction and the SPA operation cycles through a variety of modes, it is not possible to perform a full statistical correlation between the field-aligned electrons and the ICWs. When theta is small, there is good correlation provided that (1) the ULF wave is strong enough and (2) the satellite is close to the magnetic equator.

The majority of the suprathermal electrons observed have an energy of $\sim 20 \mathrm{eV}$ but the distribution extends to rather higher energies of $\sim 80 \mathrm{eV}$. Because these electrons are superimposed upon a background of photoelectrons and secondaries, it is difficult to estimate absolute fluxes; an appropriate procedure for subtracting this background is still being developed. Their pitch angle distribution is clearly peaked towards the $\mathbf{B}_{||}$direction but appears relatively broad $\left(\sim 40^{\circ}\right)$, this serves to discriminate them against ionospheric photoelectrons seen in the 'source cone' $\left(\sim 3^{\circ}\right)$.

The inferior correlation obtained with GEOS 1 $\left(\lambda_{m} \sim 15-30^{\circ}\right)$ compared with GEOS $2\left(\lambda_{m} \sim 0-3^{\circ}\right)$ can be linked to latitudinal effects for the following reasons. Using the ray tracing technique with a dipole field model, We would expect the ICWs to be reflected at $\lambda_{m} \sim 17^{\circ}$ in a as rich plasma [Rauch, 1981]. This limit may be as much as $\sim 25^{\circ}$ for the 'dayside' magnetosphere owing to the geometrical configuration of $\mathbf{B}_{\circ}$. The electrons, however, 'mirror' at greater magnetic latitudes, especially those with low pitch angle. Data from GEOS 1, e.g., those of Figure 3, show that the enhanced low-energy electron flux can persist above $\lambda_{m} \sim 25^{\circ}$ although the wave activity has decayed.

The event occurring on August 5, 1977, was different from those discussed above. The ULF wave activity is confined to frequencies above $1.5 \mathrm{~Hz}$, peaking close to the proton gyrofrequency. The enhanced electron fluxes appear as in the previous cases, but the ionospheric photoelectron masking complication makes it difficult to establish that the energy distribution is the same. The fact that this relatively unusual observation occurs when theta is small suggests that the pitch angle distribution of the accelerated thermal electrons is more sharply fieldaligned, thus producing a sampling problem with the particular detector arrangement. The associated observations of ion 'pancakes' could be similarly limited and the marginal sensitivity of SPA for such ions would also be a contributory factor. Johnson [1981] has used one hour integrations to study such ion distributions seen by GEOS 2 at the geomagnetic equator in August 1978. He finds that there is a marked preference for times within 
three hours of local noon. The energy range of these measurements (typically $20 \mathrm{eV}$ to $100 \mathrm{eV}$ ) is rather larger than those of similar distributions reported by Horwitz and Chappell [1979]. Although a plasmaspheric origin cannot be ruled out, it is possible that these are anisotropic protons, a convection remnant of the plasma sheet, which energizes a class of ULF emissions well above $f_{\mathrm{He}^{+}}$. Further discussion of this process must be left for a later paper.

\section{Summary AND CONCLUSIONS}

A good correlation between ICWs and suprathermal field-aligned electrons has been obtained when first, the ICW energy is large enough $\left(B^{2}>0.1 \gamma^{2} \mathrm{~Hz}^{-1}\right)$; second, the plasma frequency is not too large; and third, the satellite is near the geomagnetic equator.

These results are consistent with the statistical studies of the occurrence of electrostatic ELF waves associated with the ULF events [Cornilleau-Wehrlin et al., 1981] and the presence of suprathermal electrons.

The observed low-energy electron flux enhancements tend to be associated with small pitch angles, but the distributions are often broad and cannot be accurately defined by the GEOS instrumentation. Roux et al. [1982b] propose a mechanism which accounts for the preferential energization of electrons in a direction parallel to $\mathbf{B}_{0}$. Once accelerated along field lines it is suggested that the electrons are able to radiate at higher frequency, the ELF waves observed by Cornilleau-Wehrlin [1981]. Indeed the close correlation between the three processes is striking. If this suggestion proves correct then it provides a clue to the nature of the acceleration mechanism. Since the ELF waves are strongly modulated at the ICW frequency, the accelerated electrons should exhibit the same characteristic.

We are now studying possible correlations with features in the suprathermal ion distribution functions. Relatively few events of this type have been identified, but the example presented certainly promises a rewarding line of investigation.

Although the analysis of these wave-particle interactions is only at a preliminary stage, it can be seen that, by serving as a plasma laboratory, GEOS is fulfilling one of its principal scientific objectives.

Acknowledgments. The authors (AJN, JFEJ, JJS, GLW) wish to thank R. L. F. Boyd, CBE, FRS for his continued encouragement. Their research was supported by the United Kingdom Science and Engineering Research Council funding to the Mullard Space Science Laboratory. Thanks must also go to CNES Toulouse for their help in processing the ULF data and to R. Gendrin, J. L. Rauch, and P. Robert of CRPE/CNET for valuable discussions. We gratefully acknowledge the use of magnetometer data supplied by S-331 experimenters of CNR Frascati and processed by ESOC Darmstadt.

The Editor thanks M. P. Gough and another referee for their assistance in evaluating this paper.

\section{REFERENCES}

Balsiger, H., P. Eberhardt, J. Geiss, A. Ghielmetti, H. P Walker, D. T. Young, H. Loidl, and H. Rosenbauer, A satellite borne ion mass spectrometer for the energy range 0 to $16 \mathrm{keV}$, Space Sci. Insti., 2, 499-521,1976.

Cornilleau-Wehrlin, N., A new ULF modulated electrostatic wave detected in the extremely low frequency range onboard GEOS, J. Geophys. Res., 86, 1365, 1981.

Cornwall, J. M., F. V. Coroniti, and R. M. Thorne, Unified theory of SAR arc formation at the plasmapause, $J$. Geophys. Res., 76, 4428, 1971.

Horwitz, J. L., and C. R. Chappell, Observations of warm plasma in the dayside plasma trough at Geosynchronous Orbit, J. Geophys. Res., 84, 7075, 1979.

Johnson, J. F. E., Magnetospheric studies with suprathermal plasma analyzers on the ESA GEOS satellites, Ph.D. thesis, University of London, 1981.

Jones. D., Refractive index and attenuation surfaces in the vicinity of the cross-over level, Planet. Space Sci., 20, 1173-1184, 1972.

Jones, D., Introduction to the S-300 wave experiment onboard GEOS, Space Sci. Rev., 22, 327, 1978.

Leer, E., K. M. Johansen and R. Albrigtsen, Group velocity of whistlers in a two ion plasma, J. Geophys. Res., 83, 3125-3135, 1978.

Mauk, B. H., C. E. McIlwain, and R. L. McPherron, Helium cyclotron resonance within the earth's magnetosphere, Geophys. Res. Lett., 8, 103, 1981.

Mauk, B. H., and R. L. McPherron, An experimental test of the electromagnetic ion cyclotron instability within the earth's magnetosphere, Phys. Fluids, 23, 2111, 1980.

Perraut, S., R. Gendrin, P. Robert, A. Roux, C. de Villedary, and D. Jones, ULF waves observed with magnetic and electric sensors on GEOS-1, Space Sci. Rev., 22, 347, 1978.

Rauch, J. L., Etude des ondes UBF observees a bord des satellites Europeens GEOS-1 et GEOS-2, Trace de rayon dans un magnetoplasma a trois composantes, Thesis Paris VI University, 1981.

Roux, A., S. Perraut, J. L. Rauch, C. de Villedary, G. Kremser, A. Korth, and D. T. Young, Wave particle interactions near $\Omega_{\mathrm{He}}+$ observed on GEOS 1 and 2, 2, Generation of ion cyclotron waves and heating of the $\mathrm{He}^{+}$ions, J. Geophys. Res., 84, 8174, $1982 a$.

Roux, A., N. Cornilleau-Wehrlin, and J. L. Rauch, Acceleration of thermal electrons by quasi-electrostatic ULF waves, submitted to J. Geophys. Res., $1982 b$.

S-300 Experimenters, Measurements of electric and magnetic wave fields and of cold plasma parameters onboard GEOS 1 Preliminary results, Planet. Space Sci., 27, 317-339, 1979.

Smith, R. L., and N. Brice, Propagation in multi-component plasmas, J. Geophys. Res., 69, 5029-5040, 1964.

Stix, T. H., Oscillations of a cylindrical plasma, Phys. Rev. 106, 1146-1150, 1957.

Stix, T. H., The Theory of Plasma Waves, McGraw-Hill, New York, 1962.

Wrenn, G. L., J. F. E. Johnson, and J. J. Sojka, Stable pancalke distributions of low energy electrons in the plasma trough Nature, 279, 512, 1979.

Wrenn, G. L., J. F. E. Johnson, and J. J. Sojka, The suprather mal plasma analyzer on the ESA GEOS satellites, Space Sc Insti., 5, 271-293, 1981.

Young, D. T., S. Perraut, A. Roux, C. de Villedary, R. Gendrin A. Korth, G. Kremser, and D. Jones, Wave particle interac tions near $\Omega_{\mathrm{He}}+$ observed on GEOS 1 and 2, 1, Propagation 86 ion cyclotron waves in a $\mathrm{He}^{+}$rich plasma, J. Geophys. Re 6755, 1981.

(Received September 11, 1981; revised June 15, 1981; accepted July 20, 1982.) 